\title{
HIGH-ORDER ROGUE WAVES FOR THE HIROTA EQUATION
}

\author{
LINJING LI, ZHIWEI WU, LIHONG WANG, JINGSONG HE* \\ $\dagger$ Department of Mathematics, Ningbo University, Ningbo, Zhejiang 315211, P. R. China
}

\begin{abstract}
The Hirota equation is better than the nonlinear Schrödinger equation when approximating deep ocean waves. In this paper, high-order rational solutions for the Hirota equation are constructed based on the parameterized Darboux transformation. Several types of this kind of solutions are classified by their structures.
\end{abstract}

Keywords: Hirota equation, Darboux transformation, high-order rogue wave.

PACS number(s): 42.65.Tg, 47.35.Fg, 05.45Yv

\section{INTRODUCTION}

In recent decades, the rogue waves [1] (or freak waves [2]), have gained more and more interests in the study of physics. One of the reason is that such kind of waves emerges spontaneously and frequently in the ocean without any sign, "appears from nowhere and disappears without a trace [3]". Recently, besides oceanography, the rogue waves are also found in the optical systems when generating highly energetic optical pulses [4,5], as well as in the superfluids and femtosecond pulse [6 10], etc. The growing consensus, which is from the oceanic and optical rogue waves, arises from to the modulation instability(MI) 11 15 which is a key property for many integrable equations. One of the typical example is the nonlinear Schrödinger(NLS) equation

$$
i q_{t}+2|q|^{2} q+q_{x x}=0
$$

For this equation, the lower-order rogue wave has been obtained [16] with a good experimental simulation. People are more interested in the higher order waves, because its numerous and complicated properties. Some special higher order rogue waves have been constructed by Darboux transformation [17,18, and some more general cases (multi-Peregrine) were well studied 19 23]. Unlike soliton solutions siting on the zero background, these higher order rogue waves not only sit on the non-zero constant background, but lots of behaviors are quite different at points on the space-time plane 23. On the other hand, the rogue waves are the limit of a sequence of the breather solutions [18, 24]. These new studies about rogue waves enrich the conception of rogue waves and lead to understand further towards this mysterious phenomenon.

However, the phenomena of the ocean are significantly complicated, such as the depth of the sea, bottom friction, viscosity, etc, these factors all need to be taken into account when we do the simulation. Therefore we need to add higher order terms and other nonlinear effects [25,26]. The Hirota equation can be viewed as a generalization for the NLS equation, when the higher dispersion and time-delay changes is taken into account.

In dimensionless form, the Hirota equation is given by

$$
i q_{t}+\alpha\left(2|q|^{2} q+q_{x x}\right)+i \beta\left(q_{x x x}+6|q|^{2} q_{x}\right)=0, \alpha, \beta \in \mathbb{R} .
$$

*Corresonding Author: Email: hejingsong@nbu.edu.cn, Tel: 86-574-87600739, Fax: 86-574-87600744. 
The variable $t$ is the propagation variable and $x$ is the retarded time variable in a moving frame. The phase function $q$ is the envelope of the wave field. The two terms in (1.2) that enter with a real coefficient $\beta$ represent the third-order dispersion $\left(q_{x x x}\right)$ and a time-delay correction to the cubic term $\left(|q|^{2} q_{x}\right)$, respectively. We can see that

(1) $\alpha=1, \beta=0,(1.2)$ becomes the NLS equation: $i q_{t}+2|q|^{2} q+q_{x x}=0$,

(2) $\alpha=0, \beta=1,(1.2)$ becomes the modified $\mathrm{KdV}(\mathrm{mKdV})$ equation:

$q_{t}+q_{x x x}+6|q|^{2} q_{x}=0$.

In some cases, if an integrable equation is a combination of two known equations that are also integrable, the low-order solutions can be derived from solutions of those two equations. But for high-order rogue waves, it does not work. Therefore, we provide the parameterized Darboux transformation to derive the high-order rational solutions.

The organization of this paper is as follows. In section 2, based on the parameterized Darboux transformation, the general formation of the solution is given in terms of the Gram determinants. In section 3, we construct the higher order rogue waves from a periodic seed with a constant amplitude and make a classification based on their structures. We provide further discussion in section 4 .

\section{The Darboux Transformation for the Hirota equation}

In this section, we give the explicit Darboux transformation for the Hirota equation.

The Lax pair for the Hirota equation is as follows [27]:

$$
\begin{cases}\psi_{x} & =M \psi, \\ \psi_{t} & =N \psi,\end{cases}
$$

where

$$
\begin{aligned}
& \psi=\left(\begin{array}{c}
\phi_{1} \\
\phi_{2}
\end{array}\right), M=\left(\begin{array}{cc}
-i \lambda & q \\
r & i \lambda
\end{array}\right), N=V_{3} \lambda^{3}+V_{2} \lambda^{2}+V_{1} \lambda+V_{0}, \\
& V_{3}=\left(\begin{array}{cc}
-4 i \beta & 0 \\
0 & 4 i \beta
\end{array}\right), V_{2}=\left(\begin{array}{cc}
-2 i \alpha & 4 q \beta \\
4 \beta \gamma & 2 i \alpha
\end{array}\right), V_{1}=\left(\begin{array}{cc}
-2 i q r \beta & 2 i q_{x} \beta+2 q \alpha \\
-2 i r_{x} \beta+2 r \alpha & 2 i q r \beta
\end{array}\right), \\
& V_{0}=\left(\begin{array}{cc}
-i q r \alpha+\beta\left(-q r_{x}+q_{x} r\right) & i q_{x} \alpha-\beta\left(q_{x x}-2 q^{2} r\right) \\
-i r_{x} \alpha+\beta\left(-r_{x x}+2 q r^{2}\right) & i q r \alpha-\beta\left(-q r_{x}+q_{x} r\right)
\end{array}\right) .
\end{aligned}
$$

Here $\lambda$ is a spectral parameter with complex value, and $\psi$ is the eigenfunction corresponding to the $\lambda$ of the Hirota equation.

In terms of zero-curve equation $M_{t}-N_{x}+[M, N]=0$, equations for $q$ and $r$ are as follows:

$$
\left\{\begin{array}{l}
q_{t}=i \alpha\left(2|q|^{2} q+q_{x x}\right)-\beta\left(q_{x x x}+6|q|^{2} q_{x}\right), \\
r_{t}=-i \alpha\left(2|r|^{2} r+r_{x x}\right)-\beta\left(r_{x x x}+6|r|^{2} r_{x}\right),
\end{array}\right.
$$

These two equations admit the constraint

$$
r=-q^{*},
$$

here $*$ denotes the complex conjugate. After the constraint, the system (2.5) becomes one equation, which is exactly the Hirota equation.

Next, we will construct a gauge transformation

$$
\psi^{[1]}=T \psi,
$$


such that, after this gauge transformation,

$$
\begin{cases}\psi_{x}^{[1]}=M^{[1]} \psi^{[1]}, & M^{[1]}=\left(T_{x}+T M\right) T^{-1} \\ \psi_{t}^{[1]}=N^{[1]} \psi^{[1]}, & N^{[1]}=\left(T_{t}+T N\right) T^{-1}\end{cases}
$$

is solvable for $\psi^{[1]}$ and $M^{[1]}, N^{[1]}$ are of the same form as $M, N$. Moreover, we want $M^{[1]}, N^{[1]}$ also admits the constraint (2.6). Therefore, we can get new solution of the Hirota equation from one solution.

Let $\left\{\psi_{j} ; 1 \leq j \leq n\right\}$ be eigenfunctions for the Lax pair (2.1) which are corresponding to eigenvalues $\lambda_{j}, j=1,2, \ldots, n$ :

$$
\psi_{j}=\left(\begin{array}{c}
\phi_{j 1}\left(x, t, \lambda_{j}\right) \\
\phi_{j 2}\left(x, t, \lambda_{j}\right)
\end{array}\right), j=1,2, \ldots \ldots . . .
$$

Moreover, if $\psi_{j}$ is a eigenfunction for eigenvalue $\lambda_{j}$, then

$$
\psi_{j}^{\prime}:=\left(\begin{array}{c}
-\phi_{j 2}^{*} \\
\phi_{j 1}^{*}
\end{array}\right)
$$

is the eigenfunction corresponding to $\lambda_{j}^{*}$.

Choose a sequence of eigenvalues $\left\{\lambda_{j} \mid j=1,2,3, \ldots, 2 n\right\}$, such that $\lambda_{2 n-1}^{*}=\lambda_{2 n}$ and $\left\{\psi_{1}, \psi_{2}, \psi_{3}, \ldots, \psi_{2 n}\right\}$ is the corresponding eigenfunctions. We use a modified mechanism described in [18] to construct the parameterized Darboux transformation for the Hirota equation:

Theorem 1. Given $\lambda_{1} \in \mathbb{C}$, and

$$
\psi_{1}=\left(\begin{array}{c}
\phi_{11} \\
\phi_{12}
\end{array}\right)
$$

is an eigenfunction corresponding to $\lambda_{1}$ of 2.1), denote

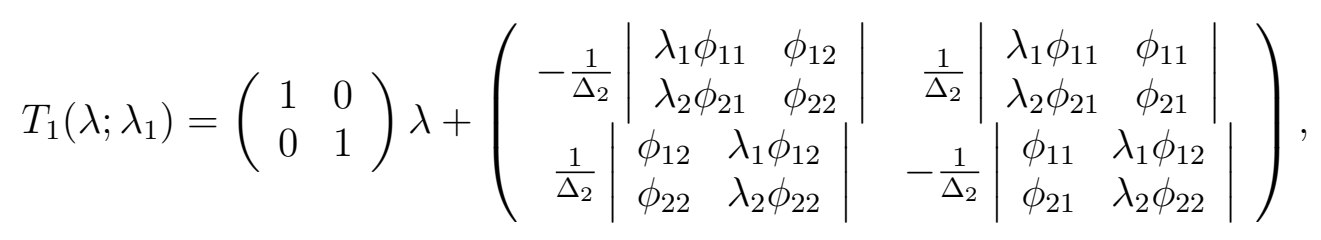

with $\Delta_{2}=\left|\begin{array}{ll}\phi_{11} & \phi_{12} \\ \phi_{21} & \phi_{22}\end{array}\right|$, then

$$
\psi^{[1]}=T_{1} \psi_{1}
$$

is again a solution of (2.1) and

$$
q_{1}=q+2 i \frac{1}{\Delta_{2}}\left|\begin{array}{cc}
\lambda_{1} \phi_{11} & \phi_{11} \\
\lambda_{2} \phi_{21} & \phi_{21}
\end{array}\right|
$$

is a new solution for the Hirota equation.

This formula is of the same form as in [18]. For high-order rational solutions $q_{n}, r_{n}$, calculation is quite cumbersome, but one can still check these equations with the aid of modern tools such as "maple" or equivalent, and also numerically.

Theorem 2 (N-fold). By applying the gauge transformation repeatedly for $N$ times, choose eigenvalues $\left\{\lambda_{1}, \lambda_{2}, \lambda_{3}, \ldots, \lambda_{2 n}\right\}$, such that $\lambda_{2 n-1}^{*}=\lambda_{2 n}, n=1,2, \ldots, N$, and $\psi_{j}$ is an eigenfunction for $\lambda_{j}$. Then we can get $N$-fold solution for the Hirota equation:

$$
q_{n}=q-2 i \frac{A_{2 n}}{B_{2 n}},
$$


where

$$
\begin{aligned}
A_{2 n} & =\left|\begin{array}{ccccccc}
\phi_{11} & \phi_{12} & \lambda_{1} \phi_{11} & \lambda_{1} \phi_{12} & \ldots & \lambda_{1}^{n-1} \phi_{11} & \lambda_{1}^{n} \phi_{11} \\
\phi_{21} & \phi_{22} & \lambda_{2} \phi_{21} & \lambda_{2} \phi_{22} & \ldots & \lambda_{2}^{n-1} \phi_{21} & \lambda_{2}^{n} \phi_{21} \\
\phi_{31} & \phi_{32} & \lambda_{3} \phi_{31} & \lambda_{3} \phi_{32} & \ldots & \lambda_{3}^{n-1} \phi_{31} & \lambda_{3}^{n} \phi_{31} \\
\phi_{41} & \phi_{42} & \lambda_{4} \phi_{41} & \lambda_{4} \phi_{42} & \ldots & \lambda_{4}^{n-1} \phi_{41} & \lambda_{4}^{n} \phi_{41} \\
\vdots & \vdots & \vdots & \vdots & \vdots & \vdots & \vdots \\
\phi_{2 n 1} & \phi_{2 n 2} & \lambda_{2 n} \phi_{2 n 1} & \lambda_{2 n} \phi_{2 n 2} & \ldots & \lambda_{2 n}^{n-1} \phi_{2 n 1} & \lambda_{2 n}^{n} \phi_{2 n 1}
\end{array}\right| \\
B_{2 n}= & \left|\begin{array}{ccccccc}
\phi_{11} & \phi_{12} & \lambda_{1} \phi_{11} & \lambda_{1} \phi_{12} & \ldots & \lambda_{1}^{n-1} \phi_{11} & \lambda_{1}^{n-1} \phi_{12} \\
\phi_{21} & \phi_{22} & \lambda_{2} \phi_{21} & \lambda_{2} \phi_{22} & \ldots & \lambda_{2}^{n-1} \phi_{21} & \lambda_{2}^{n-1} \phi_{22} \\
\phi_{31} & \phi_{32} & \lambda_{3} \phi_{31} & \lambda_{3} \phi_{32} & \ldots & \lambda_{3}^{n-1} \phi_{31} & \lambda_{3}^{n-1} \phi_{32} \\
\phi_{41} & \phi_{42} & \lambda_{4} \phi_{41} & \lambda_{4} \phi_{42} & \ldots & \lambda_{4}^{n-1} \phi_{41} & \lambda_{4}^{n-1} \phi_{42} \\
\vdots & \vdots & \vdots & \vdots & \vdots & \vdots & \vdots \\
\phi_{2 n 1} & \phi_{2 n 2} & \lambda_{2 n} \phi_{2 n 1} & \lambda_{2 n} \phi_{2 n 2} & \ldots & \lambda_{2 n}^{n-1} \phi_{2 n 1} & \lambda_{2 n}^{n-1} \phi_{2 n 2}
\end{array}\right| .
\end{aligned}
$$

As a consequence, high-order rogue waves for the NLS equation and the mKdV equation can be derived.

\section{The High-ORder Rogue WAVES}

In this section, we give high-order rogue waves for the Hirota equation from non-zero seeds. By using the principle of the superposition of the linear differential equation, we can get the new eigenfunction corresponding to $\lambda_{j}$ :

with

$$
\begin{gathered}
\psi_{j}=\left(\begin{array}{c}
\varphi_{j 1} \\
\varphi_{j 2}
\end{array}\right) \\
\varphi_{j 1}=d_{1} \phi_{j 1}+d_{2} \phi_{j 2}, \varphi_{j 2}=-d_{1} \phi_{j 2}^{*}+d_{2} \phi_{j 1}^{*},
\end{gathered}
$$

$$
\begin{gathered}
d_{1}=e^{i c_{1}\left(s_{0}+s_{1} \varepsilon+s_{2} \varepsilon^{2}+\ldots+s_{n-1} \varepsilon^{n-1}\right)}, d_{2}=e^{-i c_{1}\left(s_{0}+s_{1} \varepsilon+s_{2} \varepsilon^{2}+\ldots+s_{n-1} \varepsilon^{n-1}\right)}, \\
\phi_{11}=c e^{i\left[\left(\frac{1}{2} a+c_{1}\right) x+\left(\frac{1}{2} b+2 c_{1} c_{2}\right) t\right]}, \phi_{12}=i\left(\frac{1}{2} a+\lambda_{j}+c_{1}\right) e^{i\left[\left(-\frac{1}{2} a+c_{1}\right) x+\left(-\frac{1}{2} b+2 c_{1} c_{2}\right) t\right]}, \\
c_{1}=\frac{1}{2} \sqrt{a^{2}+4 c^{2}+4 \lambda_{j} a+4 \lambda_{j}^{2}}, \quad c_{2}=\alpha \lambda_{j}+2 \beta \lambda_{j}^{2}-\frac{1}{2} a \alpha-\beta c^{2}+\frac{1}{2} \beta a^{2}-\lambda_{j} \alpha \beta .
\end{gathered}
$$

Here $a, b, c, s_{i}, i=0,1,2, \ldots$ are the constants, $\varepsilon$ is infinitesimal, $\psi_{1}=\left(\phi_{11}, \phi_{12}\right)^{T}$ is the solution of (2.1) for the non-zero seed $q=c e^{i \rho}, \rho=a x+b t, b=\alpha\left(2 c^{2}-a^{2}\right)+\beta\left(a^{3}-6 a c^{2}\right)$.

It is well known that high-order rogue waves can be obtained by the Taylor expansion of the breather solutions. In [18], the scheme for nonlinear schrödinger equation is established. Due to the complication and tediousness of the explicit formula, we describe the high-order rogue waves by their structures, explicit formula for solution $q_{1}$ and $q_{2}$ will be given in the Appendix. Moreover, in the rest of this paper, we will dedicate to the classification of rational solutions.

In our classification, we can see that by choosing different values for parameters $d_{1}$ and $d_{2}$ different types of solutions are obtained.

(1) When $d_{1}=d_{2}=1$ (after expansion), rational solutions of any order $n$ have similar structure.

(a) There are $\frac{n(n+1)}{2}-1$ local maximum in each side of the line $t=0$.

(b) Starting from $-\infty$, before the central high amplitude (the global maximum), there is a sequence of peaks with increasing height. In detail, the number of first peaks is $n$, then there is a row of $n-1$ peaks and so on. 
(c) The structure is symmetric with respect to time $t$.

We called this type of rogue wave the fundamental pattern (same as in [18] Figure 1).

(2) Ring structure.

In (3.1), when $n \geq 3$ and the principle coefficient for order $n$ rational solution $s_{n-1} \gg$ 1 , while other coefficients $s_{i}$ are all zero. The structure for rational solutions consists of two parts. The outer circular shell consists of $2 n-1$ first order rational solutions, while the center is an order $(n-2)$ rational solution of fundamental pattern. This structure is similar to the "wave clusters" in [28]. Figure 2 give a visual description for the ring structure.

(3) Triangular structure.

Another typical type of rational solutions can be obtained by choosing the first nontrivial coefficient $s_{1} \gg 1$, while others are all zero. The order $n$ rational solutions have a structure of equilateral triangular type with $n$ peaks in each edge. Each single peak is of the same height and the total number of peaks is $\frac{n(n+1)}{2}$ (Figure 3).

Remark 3.1. When $n=2$, the ring structure and triangular structure have the same shape: there is a single triangular with $3=3 n-3$ peaks in the triangular structure, and in the ring structure, since $n-2=0$, there is no basic mode in the middle and the outer ring consists of $3=2 n-1$ peaks.

These three types of structures listed above are the basic scheme for high-order rogue waves. Another interesting fact worth emphasized here is that: high-order rogue waves are usually the combination of these three types: "fundamental pattern", "ring" structure and "triangular" structure. In the rest part of this paper, we will discuss these combination types and their dependence on the free parameters.

(1) As we mentioned before, the principle coefficient " $s_{n-1}$ " determined the ring structure. Based on this, if only the preceeding coefficient " $s_{n-2}$ " is non zero, the high-order rogue waves have a double-ring structure. More explicitly, there are two rings with $2 n-3$ single peaks in each and one $(n-4)$ basic mode in the middle (Figure 4 ). For example, (c) shows this type of combination for $\left|q_{6}\right|^{2}$. There are two outer ring structures with one 2-nd order basic mode in the center(Figure $1(\mathrm{~b})$ ), and each individual ring is consisted of 9 single peaks. When $n=4$, there is no fundamental pattern since $n-4=0$ (a).

(2) Triple-ring structure is determined by the coefficient $s_{n-3}$. When $s_{n-3} \neq 0$, the order $n$ rogue wave has a triple ring structure with $n$ single peaks in the outer circular and an order $n-1$ double ring structure in the middle (Figure 5).

(3) If $s_{1}$ and $s_{n-1} \neq 0$, the phase space of high-order waves can be decomposed into two parts. The outer circular shell has $2 n-1$ single peaks while the center is an order $n-2$ rogue wave with triangular structure (Figure 6).

(4) If both $s_{n-1}$ and $s_{n-2}$ are non zero, then the order $n$ rogue wave is a composition of order $n$ and order $n-2$ ring structure such that the outer cell has $2 n-1$ single peaks. And the inside is an order $(n-2)$ ring structures (Figure 7$)$. Here we need to point out that although the shape is similar to double-ring structure described above, they are of different pattern in combination. In double-ring structure, both two outer circulars have $2 n-3$ single peaks in each. While here, the outer cells have $2 n-1$ and $2 n-5$ single peaks in each respectively.

(5) Other parameters also play an important role in determining the structure of highorder rogue waves. In other words, by choosing the parameter properly, we can get more complicated structures. Here we give one more example. It is a composition of 
order $n$ ring structure and $n-2$ double-ring structure (Figure 8). Moreover, it is a complete decomposition in the ring structure. That is, each local maximum is of the same value and there is no fundamental pattern.

Remark 3.2. There are several coincides between different patterns, that is due to the special value of $n$. For example, when $n=4$, then $n-3=1$, therefore, the triple-ring structure of the same shape as ring structure is for triangular structure.

Beside different structures, there are some properties that are preserved by all types of rogue wave solutions. First, the total number of peaks in order $n$ solutions is $\frac{n(n+1)}{2}$. Secondly, the structures depend on every parameter. The principle coefficient $s_{n-1}$ leads to the ring structure. The first nontrivial coefficient $s_{1}$ leads to triangular solutions. They are very similar to the rational solutions of the NLS equation apart from the finite tilt with regard to the axes. We find that the main effect of the third-order dispersion and a time-delay correction terms added to the NLS equation is to introduce a "tilt" to the NLS equation solutions [29], namely, the tilt is related to the finite value of parameter $\beta$, because if we set $\beta=0$ the tilt is zero. We can shorten the length of the "ridge" by taking the value of $a$ properly, moreover, of interest it is to find that on the central line where $t=0$ the shape is independent of $\beta$ and $a$. That is, the adding the $\mathrm{mKdV}$ terms to the NLS equation do not have any impact in the figures of the rogue waves at the $t=0$ where the figures have its maximum. Consequently, the overall maximum value of the amplitude figure is $q_{j}(0,0)$ for any value of $\beta$, which is indeed the same as the NLS equation.

\section{Conclusions And Discussions}

In this paper, we use the parameterized Darboux transformation to construct the high-order rational solutions for the Hirota equation. Based on the theoretical result, we classify the highorder rogue waves with respect to their intrinsic structure. We find out that there are three principle types, basic mode, ring structure, and triangular structure. Moreover, the composition of the three principle structures are held by high-order rogue waves. As we mentioned in the very beginning, the Hirota equation is a generalized NLS equation. The ring structures obtained in this paper are similar to the "atom" in Ref [28]. This explains the generalization in terms of the solution. On the other hand, by changing the free parameters in the Darboux transformation we can get more complicated structures. It would be promising to ask whether there are some analogues between high-order rogue waves of different integrable equations? Also, it is essential to find more conserved properties for such kind of solutions. All of these will help us in a better understanding in the deep ocean wave.

On the other hand, the most remarkable feature of this equation is that it includes both the NLS equation by restricting the parameter $\beta=0$ and the mKdV equation by restricting the parameter $\alpha=0$. Namely, by taking the different values of the parameters, we can also obtain high-order rogue waves of the NLS equation and mKdV equation, with regard to this point, we shall give the detailed describing in other papers respectively.

\section{Acknowledgments}

This work is supported by the NSF of China under Grant No.11271210, No.10971109 and K. C. Wong Magna Fund in Ningbo University. Jingsong He is also supported by Natural Science Foundation of Ningbo under Grant No. 2011A610179. The authors appreciate the helpful suggestions of reviewers. 


\section{REFERENCES}

[1] Müller. P., Garrett. Ch., Osborne. A., Rogue waves: in: The fourteenth 'Aha Huliko' a Hawaiian Winter Workshop. Oceanography, 18,66-75(2005).

[2] L Draper, Freak ocean waves, Mar. Obs., 35,193-195(1965).

[3] N. Akhmediev, A. Ankiewicz, and M.Taki, Waves that appear from nowhere and disappear without a trace, Phys. Lett. A., 373, 675-678(2009).

[4] A. Hasegawa and W. F. Brinkman, Tunable coherent IR and FIR sources utilizing modulational instability, IEEE J., 16, 694-697(1980).

[5] D. Anderson and M. Lisak, Modulational instability of coherent optical fiber transmission signals, Opt. Lett., 9, 468-470(1984).

[6] N. N. Akhmediev, V. I. Korneev, and N. V. Mitskevich, Moduation instability of a continuous signal in an optical fiber taking into accout third-order dispersion, Radiophysics and quantum electronics., 33, 95-100(1990).

[7] A. Hasegawa, Generation of a train of soliton pulses by induced modulational instability in optical fibers, Opt. Lett., 9, 288-290(1984).

[8] K. Tai, J. L. Tomita, J. L. Jewell, and A. Hasegawa, Generation of subpicosecond solitonlike optical pulses at 0.3 THZ repetion rate by induced modulatinal instability, Appl. Phys. Lett., 49, 236-238(1986).

[9] K. Tai, A. Hasegawa, and A. Tomita, Observation of modulational instability in optical fibers, Phys. Rev. Lett., 56, 135-138(1986).

[10] M. J. Potasek, Experimental and numerical results of optical subpulse formation of long optical pulses in monomode fibers, Opt. Lett., 12, 717-719(1987).

[11] C. Garrett and J. Gemmrich, Rogue waves, Phys. Today 62,62-63(2009).

[12] M. Onorato, T. Waseda, A. Toffoli, L. Cavaleri, O. Gramstad, P. A. E. M. Janssen, T. Kinoshita, J. Monbaliu, N. Mori, A. R. Osborne, M. Serio, C. T. Stansberg, H. Tamura, and K. Trulsen, Statistical properties of directional ocean waves: The role of the modulational instability in the formation of extreme events, Phys. Rev. Lett. 102, 114502 (2009).

[13] J. M. Dudley, G. Genty, F. Dias, B. Kibler, and N. Akhmediev, Modulation instability, Akhmediev Breathers and continuous wave supercontinuum generation, Opt. Express 17, 21497-21508(2009).

[14] V. A. Vysloukh and N. A. Sukhotskova, Effect of third-order dispersion on the generation of a sequence of picosecond pulses in fiber-optic waveguides due to self-modulation instability, Kvantovaya Elektron., 14, 2371-2374(1987).

[15] N. N. Akhmediev, V. I. Korneev, and N. V. Mitskevich, N-modulational signals in a single-mode optical fiber with allowance for nonlinearity, Zh. Eksp. Teor. Fiz., 94, 159-170(1988).

[16] Peregrine, D. H., Water waves, nonlinear Schrödinger equations and their solutions, J. Aust. Math. Soc. B, 25, 16-43(1983).

[17] Akhmediev. N, Ankiewicz. A, Soto-Crespo, J. M., Rogue waves and rational solutions of the nonlinear Schrödinger equations, Phys. Rev. E, 80. 026601(2009).

[18] Jingsong He, Hongrui Zhang, Lihong Wang, Porsezian. K. and Fokas. A. S., A generating mechanism of the higher order rogue waves, (submitted)(2012) arXiv:1209.3742 3 .

[19] Dubard, P., Gaillard, P., Klein, C. Matveev, V. B. On multi-rogue wave solutions of the NLS equation and positon solutions of the KdV equation. Eur. Phys. J. Spec. Top., 185, 247C258(2010).

[20] Dubard. P., Matveev. V. B., Multi-rogue waves solutions to the focusing NLS equation and the KP-I equation, Nat. Hazards Earth. Syst. Sci., 11, 667-672(2011) .

[21] Gaillard. P., Families of quasi-rational solutions of the NLS equation and multi-rogue waves, J. Phys. A: Math. Theor., 44, 435204(2011).

[22] Yasuhiro Ohta and Jianke Yang, General high-order rogue waves and their dynamics in the nonlinear Schrödinger equation, Proc. R. Soc. A, 468, 1716-1740(2012).

[23] Ankiewicz, A., Kedziora. D. J., Akhmediev. N., Rogue wave triplets, Phys. Lett. A., 375, 2782-2785(2011).

[24] Simon Clarke, Roger Grimshaw, and Peter Miller, On the generation of solitons and breathers in the modified Korteweg-de Vries equation, Chaos, 10, 383-392(2000).

[25] K. Ohkuma, H. Yoshi, H. Ichikawa, and Y. Abe, Soliton propagation along optical fibers, Opt. Lett., 12, 516-518(1987).

[26] D. N. Christodoulides and R. H. Joseph, Femtosecond solitary waves in optical fibers-beyond the slowly varying envelope approximation, Appl. Phys. Lett., 47, 76-78(1985). 
[27] Yongsheng Tao, Jingsong He, Multisolitons, breathers, and rogue waves for the Hirota equation generated by the Darboux transformation, Phys. Rev. E., 85, 026601(2012).

[28] David J. Kedziora, Adrian Ankiewicz, and Nail Akhmediev, Circular rogue wave clusters, Phys. Rev. E., 84, 056611(2011).

[29] N. Akhmediev, V. I. Korneev, and N. V. Mitskevich, Izv. Vyssh. Uchebn. Zaved.,Radiofiz., Modulation instability of a continuous signal in an optical fiber taking into accout third-order dispersion, Physics and quantum electronics, 33, 95-100 (1990) [Radiofiz. 33, 111-117 (1990)].

\section{Appendix}

The formulas for $q_{1}, q_{2}$ are given under the condition $s_{0}=0, \alpha=10, \beta=1, a=0, c=1$ and $\alpha=5, \beta=1, s_{0}=0, a=3.88, c=1$ respectively.

$$
\begin{aligned}
& q_{1}=e^{20 i t} \frac{3-1744 t^{2}+48 t x+160 i t-4 x^{2}}{1+4 x^{2}+1744 t^{2}-48 t x} \\
& q_{2}=\mathrm{e}^{i(3.88 x-30.140928 t)} \frac{\Delta_{1}}{\Delta_{2}} \\
& \Delta_{1}=45+351839417.0 t^{6}-252396.4448 t^{2} x^{2}+33987.4508 t^{2} x^{4} \\
& +139.4688 x^{5} t+4344315.56 t^{5} x+49254.311 x^{3} t^{3} \\
& +1326.7968 x^{3} t+5998449.26 t^{4} x^{2}-996552.958 x t^{3} \\
& +3325.2480 t x+1307537.37 i t^{4} x-127944.917 i t^{2} x \\
& -4780.80 i t+7408.583 i t^{2} x^{3}+1802722.57 i t^{3} x^{2} \\
& +144 i s_{1}-180 x^{2}-72088.2566 t^{2}+404211.06 i t^{3} \\
& +158844096.7 i t^{5}-16750593.96 t^{4}+1912.32 t s_{1} \\
& -144 x^{4}+5556.437 t^{2} x s_{1}+7649.28 t x^{2} s_{1}+144 s_{1}{ }^{2} \\
& +418.4064 i t x s_{1}+5099.52 i t x^{4}-7649.28 i t x^{2}+64 x^{6} \\
& -448662.55 t^{3} s_{1}-101506.4548 i t^{2} s_{1}+576 i s_{1} x^{2} \\
& \Delta_{2}=9+351839351.0 t^{6}-49079.6020 t^{2} x^{2}+33987.4508 t^{2} x^{4} \\
& -5736.96 t s_{1}-448662.552 t^{3} s_{1}+5998449.36 t^{4} x^{2} \\
& +4344308.56 t^{5} x+49254.311 x^{3} t^{3}+1605.7344 x^{3} t \\
& +139.4688 x^{5} t-848937.199 x t^{3}-1073.5488 t x \\
& +108 x^{2}+78649.7667 t^{2}+48 x^{4}+13134403.21 t^{4} \\
& +5556.437 t^{2} x s_{1}+7649.28 t x^{2} s_{1}+64 x^{6}+144 s_{1}{ }^{2}
\end{aligned}
$$

On the other hand, we also give the formulas for $q_{2}$ under the condition $\alpha=5, \beta=1, s_{1}=$ $0, a=3.88, c=1$. 


$$
\begin{aligned}
& q_{2}=\mathrm{e}^{i(3.88 x-30.140928 t)} \frac{\Delta_{3}}{\Delta_{4}} \\
& \Delta_{3}=45+5099.52 i t x^{4}-7649.28 i t x^{2}+64 x^{6}-144 x^{4} \\
& -180 x^{2}+351839311.0 t^{6}-16750593.36 t^{4} \\
& -72088.2566 t^{2}+404211.06 i t^{3}+158844096.7 i t^{5} \\
& +5998449.06 t^{4} x^{2}+33987.4508 t^{2} x^{4}-252396.4448 t^{2} x^{2} \\
& -996552.948 t^{3} x+49254.311 t^{3} x^{3}+3325.2480 t x \\
& +139.4688 t x^{5}+4344348.56 t^{5} x+1326.7968 t x^{3} \\
& -4780.80 i t+7408.583 i t^{2} x^{3}+1307537.37 i t^{4} x \\
& -127944.917 i t^{2} x+1802722.57 i t^{3} x^{2}+49254.311 s_{0}{ }^{3} t^{3} \\
& -996552.928 s_{0} t^{3}+1326.7968 s_{0}{ }^{3} t+139.4688 s_{0}{ }^{5} t \\
& +5998446.04 t^{4} s_{0}{ }^{2}+33987.4508 s_{0}{ }^{4} t^{2}+3325.2480 s_{0} t \\
& -252396.4448 s_{0}^{2} t^{2}-576 x s_{0}{ }^{3}+384 x^{5} s_{0}+960 x^{4} s_{0}{ }^{2} \\
& +1280 x^{3} s_{0}{ }^{3}-864 x^{2} s_{0}{ }^{2}+384 s_{0}{ }^{5} x+960 s_{0}{ }^{4} x^{2} \\
& +4344312.56 t^{5} s_{0}-15298.56 i s_{0} t x+30597.12 i s_{0}{ }^{2} t x^{2} \\
& +20398.08 i s_{0} t x^{3}+20398.08 i s_{0}{ }^{3} t x+22225.748 i s_{0}{ }^{2} t^{2} x \\
& +22225.748 i t^{2} x^{2} s_{0}+3605445.03 i t^{3} x s_{0}-180 s_{0}{ }^{2}-144 s_{0}{ }^{4} \\
& -504792.8896 t^{2} s_{0} x+11996906.61 t^{4} s_{0} x+135949.802 t^{2} x^{3} s_{0} \\
& +203924.713 t^{2} x^{2} s_{0}{ }^{2}+135949.802 s_{0}{ }^{3} t^{2} x+697.3440 s_{0}{ }^{4} t x \\
& +1394.6880 s_{0}{ }^{3} t x^{2}+697.3440 x^{4} s_{0} t+1394.6880 x^{3} s_{0}{ }^{2} t \\
& +3980.3904 x^{2} s_{0} t+3980.3904 x s_{0}^{2} t+147763.18 t^{3} s_{0}{ }^{2} x \\
& +147763.15 t^{3} s_{0} x^{2}+7408.583 i s_{0}{ }^{3} t^{2}+1307537.37 i s_{0} t^{4} \\
& -127944.917 i s_{0} t^{2}+1802722.57 i s_{0}{ }^{2} t^{3}-360 s_{0} x \\
& +64 s_{0}{ }^{6}+5099.52 i s_{0}{ }^{4} t-7649.28 i s_{0}{ }^{2} t-576 x^{3} s_{0} \\
& \Delta_{4}=9+64 x^{6}+48 x^{4}+108 x^{2}+351839241.0 t^{6} \\
& +13134403.54 t^{4}+78649.7667 t^{2}+5998445.94 t^{4} x^{2} \\
& +33987.4508 t^{2} x^{4}-49079.6020 t^{2} x^{2}-848937.199 t^{3} x \\
& +49254.351 t^{3} x^{3}-1073.5488 t x+139.4688 t x^{5} \\
& +4344312.56 t^{5} x+1605.7344 t x^{3}+49254.311 s_{0}{ }^{3} t^{3} \\
& -848937.199 s_{0} t^{3}+1605.7344 s_{0}{ }^{3} t+139.4688 s_{0}{ }^{5} t \\
& +5998445.94 t^{4} s_{0}{ }^{2}+33987.4508 s_{0}{ }^{4} t^{2}-1073.5488 s_{0} t \\
& -49079.6018 s_{0}{ }^{2} t^{2}+192 x s_{0}{ }^{3}+384 x^{5} s_{0}+960 x^{4} s_{0}{ }^{2} \\
& +1280 x^{3} s_{0}{ }^{3}+288 x^{2} s_{0}{ }^{2}+384 s_{0}{ }^{5} x+960 s_{0}{ }^{4} x^{2}+192 x^{3} s_{0}
\end{aligned}
$$




$$
\begin{aligned}
& +216 s_{0} x+4344308.56 t^{5} s_{0}+108 s_{0}{ }^{2}+48 s_{0}{ }^{4}+64 s_{0}{ }^{6} \\
& -98159.2036 t^{2} s_{0} x+11996906.81 t^{4} s_{0} x+135949.802 t^{2} x^{3} s_{0} \\
& +203924.713 t^{2} x^{2} s_{0}{ }^{2}+135949.802 s_{0}{ }^{3} t^{2} x+697.3440 s_{0}{ }^{4} t x \\
& +1394.6880 s_{0}{ }^{3} t x^{2}+697.3440 x^{4} s_{0} t+1394.6880 x^{3} s_{0}{ }^{2} t \\
& +4817.2032 x^{2} s_{0} t+4817.2032 x s_{0}{ }^{2} t+147763.15 t^{3} s_{0}{ }^{2} x \\
& +147763.15 t^{3} s_{0} x^{2}
\end{aligned}
$$
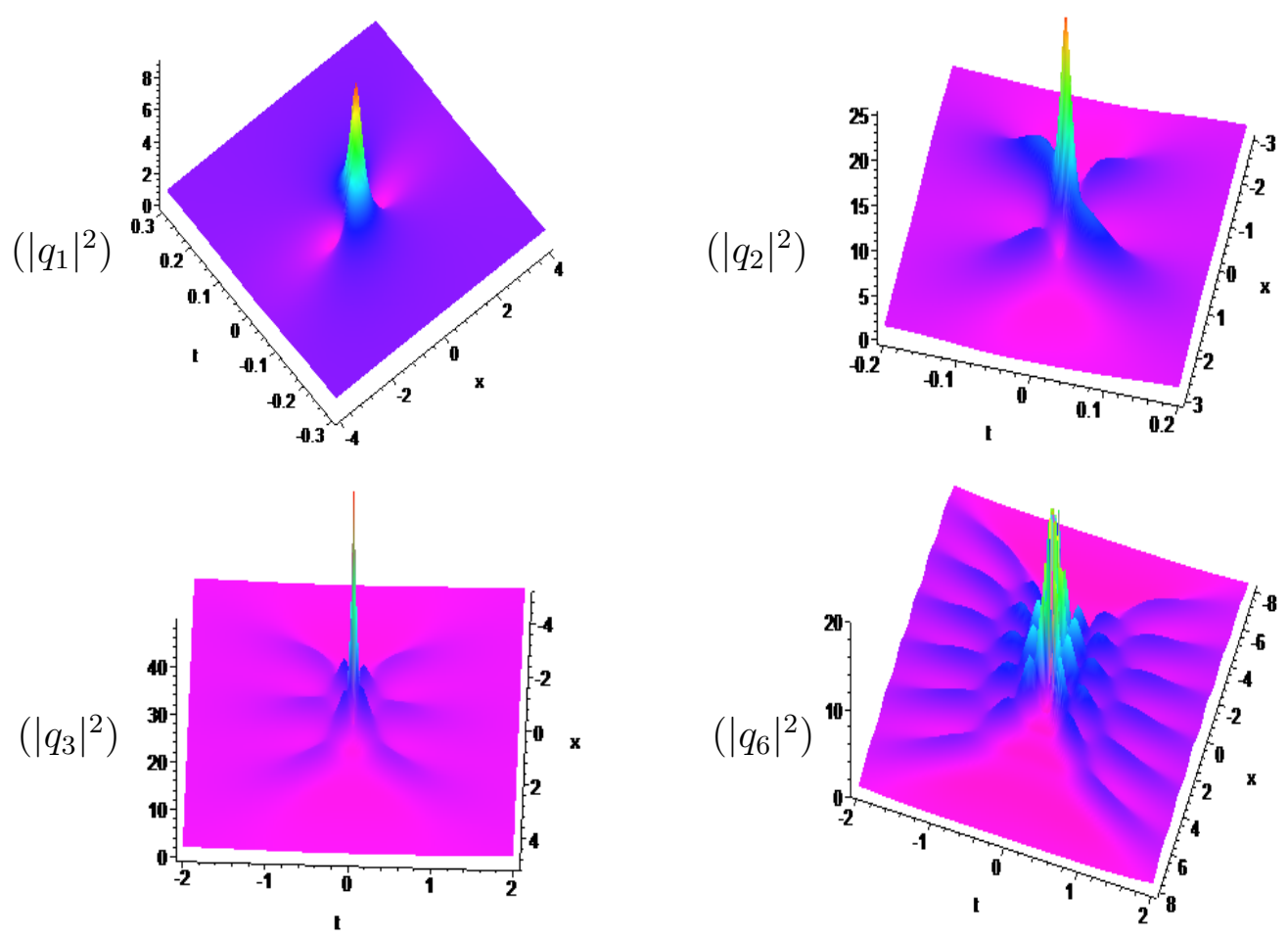

Figure 1. Higher order rogue waves with zero shifts (fundamental pattern), i.e., (a) $n=$ $1, \alpha=10, \beta=1, a=0, c=1, s_{0}=0$. (b) $n=2, \alpha=5, \beta=1, a=3.88, c=1, s_{0}=0, s_{1}=0$. (c) $n=3, \alpha=1, \beta=\frac{1}{50}, a=0, c=1, s_{0}=0, s_{1}=0, s_{2}=0$. (d) $n=6, \alpha=1, \beta=\frac{1}{50}, a=$ $0, c=1, s_{0}=0, s_{1}=0, s_{2}=0, s_{3}=0, s_{4}=0, s_{5}=0$. Here in the figure of $\left|q_{6}\right|^{2}$, to show the lower peaks clearly, the whole amplitude of the maximum (central peak) is cut. The maximum is 169 (that is $\left.(2 n+1)^{2}\right)$. 

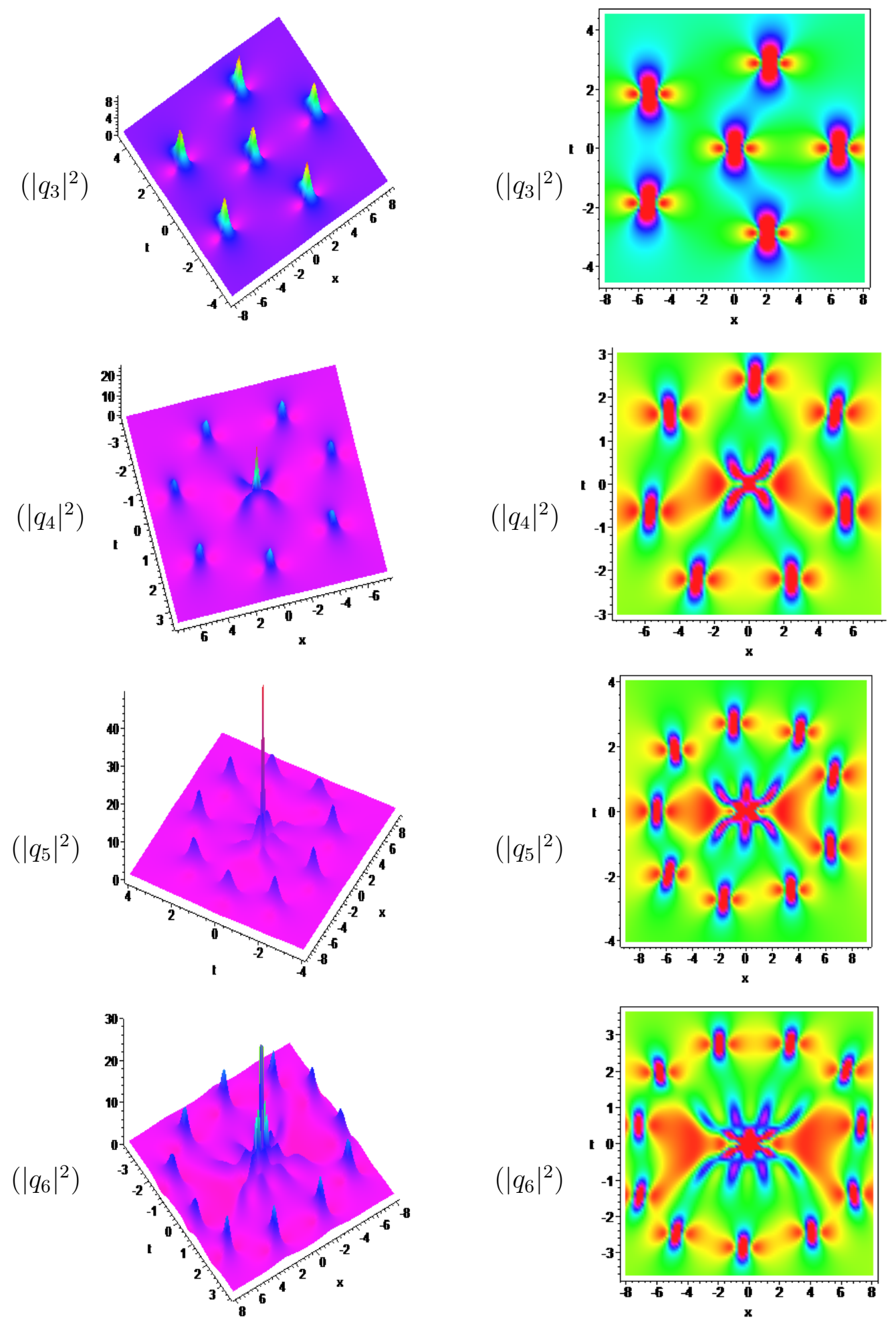

Figure 2. Single ring structure of $\left|q_{n}\right|^{2}$ (left) and the corresponding density plot (right) under the condition (a) $n=3, \alpha=1, \beta=\frac{1}{50}, a=-\frac{1}{20}, c=1, s_{0}=0, s_{1}=0, s_{2}=1000$. The trajectory is a pentagon; (b) $n=4, \alpha=1, \beta=\frac{1}{50}, a=0, c=1, s_{0}=0, s_{1}=0, s_{2}=0, s_{3}=$ 1000; (c) $n=5, \alpha=1, \beta=\frac{1}{50}, a=0, c=1, s_{0}=0, s_{1}=0, s_{2}=0, s_{3}=0, s_{4}=4500$; (d) $n=6, \alpha=1, \beta=\frac{1}{50}, a=0, c=1, s_{0}=0, s_{1}=0, s_{2}=0, s_{3}=0, s_{4}=0, s_{5}=10000$. Here in the figure of $\left|q_{6}\right|^{2}$, to show the lower peaks clearly, the whole amplitude of the maximum (central peak) is cut. The maximum of the inner order 4 rogue wave is 81 . 

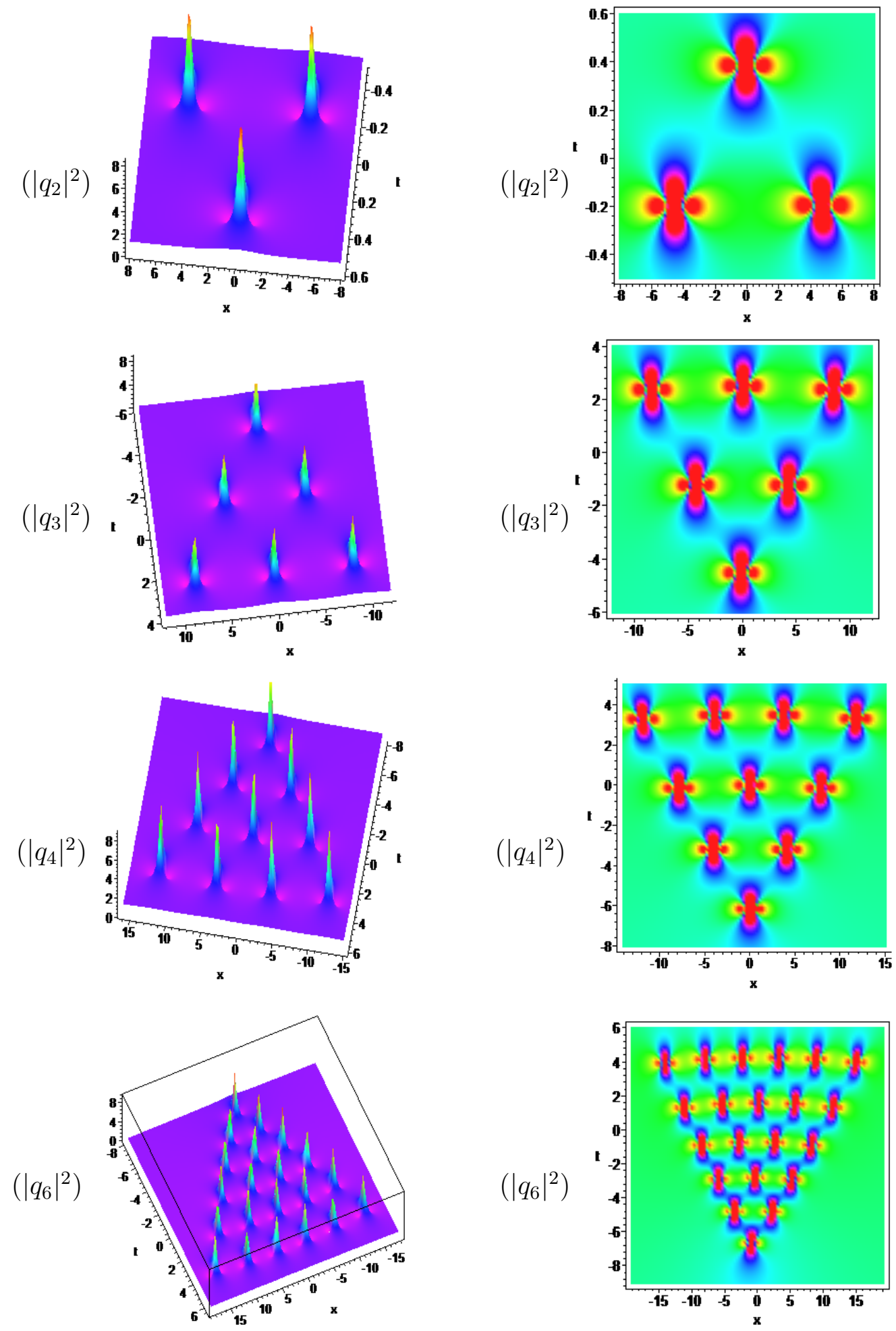

Figure 3. Triangular structure of $\left|q_{n}\right|^{2}$ (a) $n=2, \alpha=5, \beta=1, a=3.88, c=1, s_{0}=$ $0, s_{1}=100$; (b) $n=3, \alpha=1, \beta=\frac{1}{50}, a=-\frac{1}{20}, c=1, s_{0}=0, s_{1}=100, s_{2}=0$; (c) $n=4, \alpha=1, \beta=\frac{1}{50}, a=-\frac{1}{15}, c=1, s_{0}=0, s_{1}=100, s_{2}=0, s_{3}=0$; (d) $n=6, \alpha=1, \beta=$ $\frac{1}{50}, a=0, c=1, s_{0}=0, s_{1}=50, s_{2}=0, s_{3}=0, s_{4}=0, s_{5}=0$. 

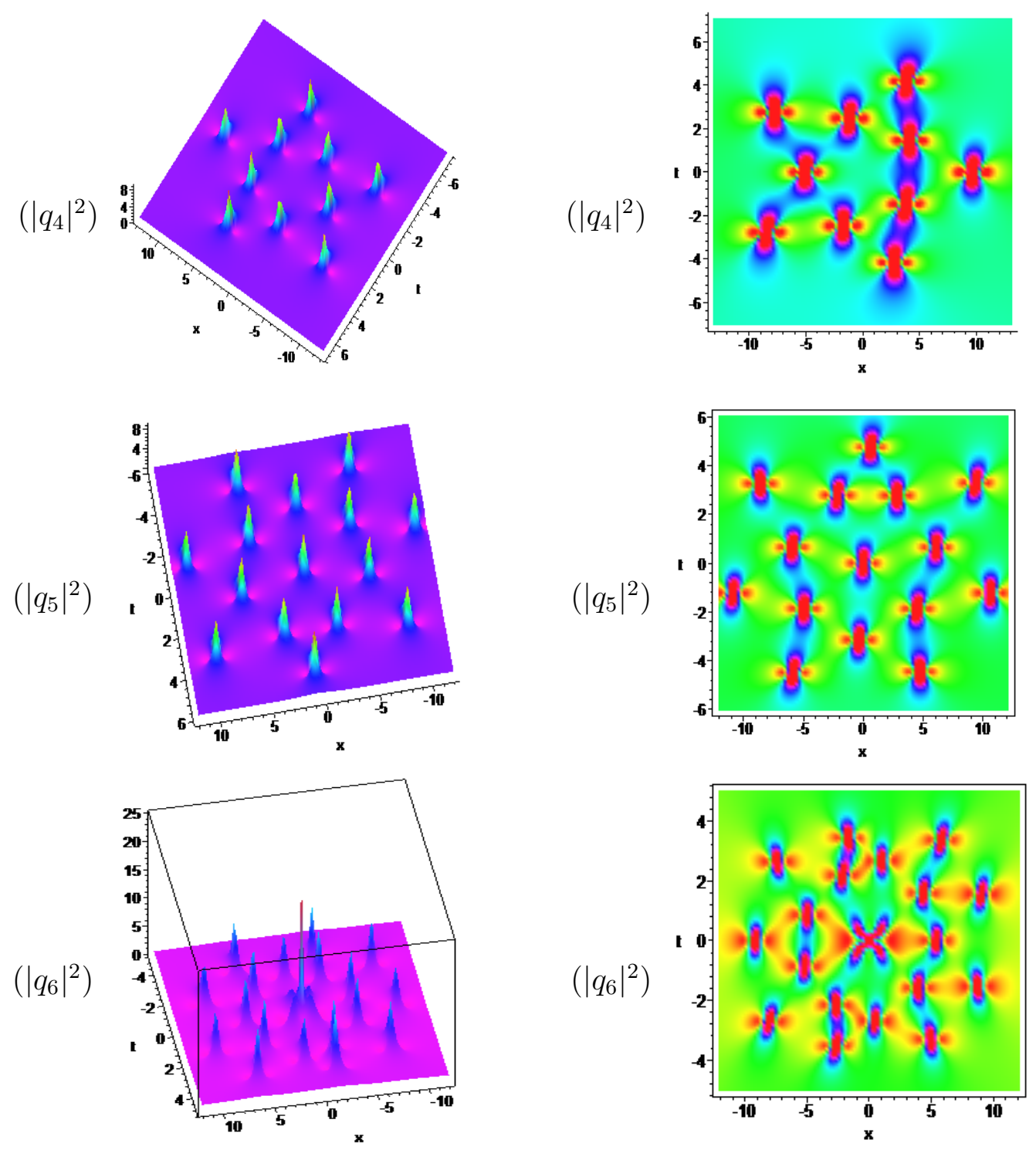

Figure 4. The decomposition of $\left|q_{n}\right|^{2}$ into double-ring structure (a) $n=4, \alpha=1, \beta=$ $\frac{1}{50}, a=0, c=1, s_{0}=0, s_{1}=0, s_{2}=1000, s_{3}=0$; (b) $n=5, \alpha=1, \beta=\frac{1}{50}, a=0, c=1, s_{0}=$ $0, s_{1}=0, s_{2}=0, s_{3}=10000, s_{4}=0$; (c) $n=6, \alpha=1, \beta=\frac{1}{50}, a=0, c=1, s_{0}=0, s_{1}=$ $0, s_{2}=0, s_{3}=0, s_{4}=6000, s_{5}=0$. 

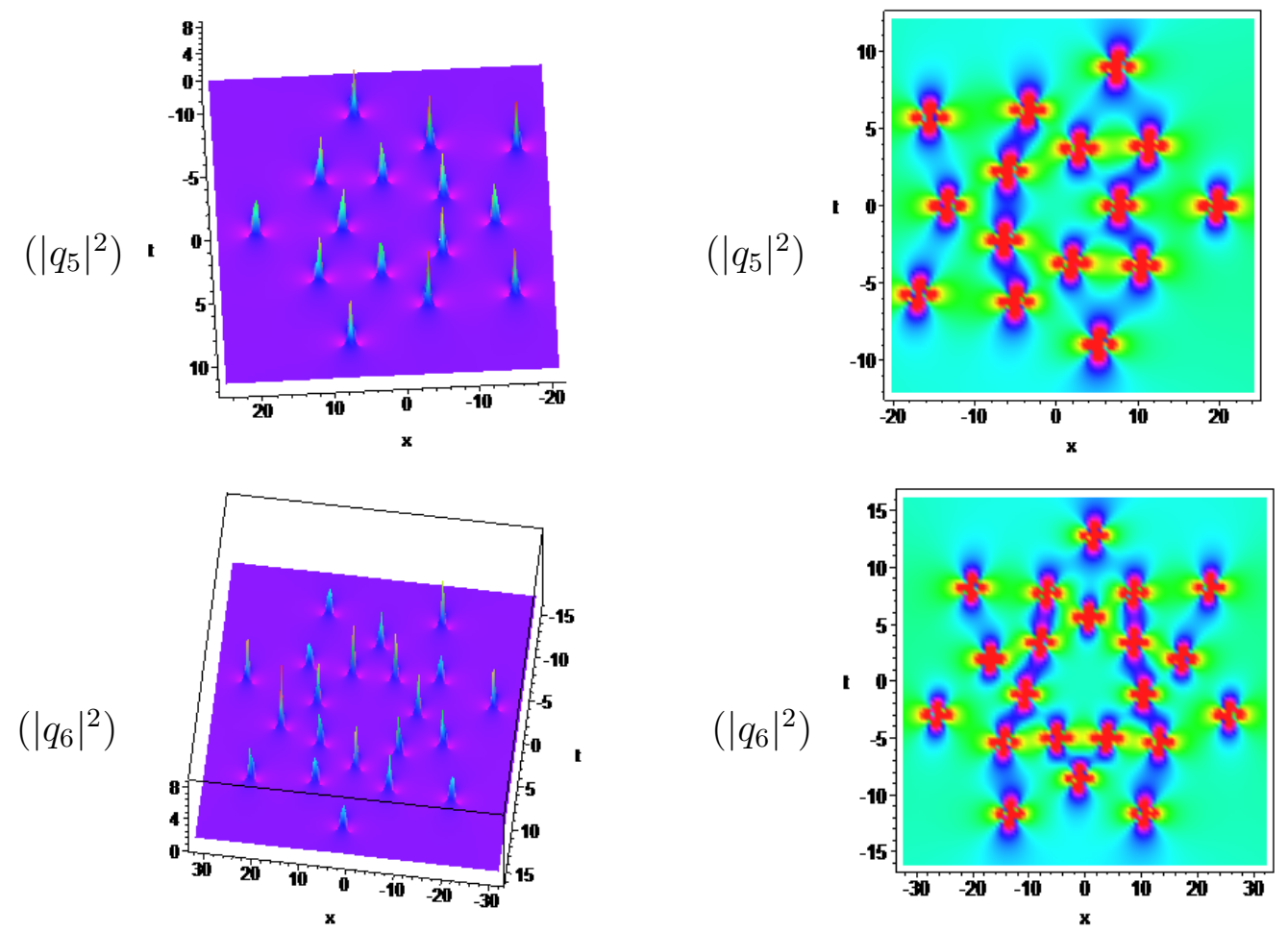

Figure 5. Triple-ring structure of $\left|q_{n}\right|^{2}$ (a) $n=5, \alpha=1, \beta=\frac{1}{50}, a=0, c=1, s_{0}=0, s_{1}=$ $0, s_{2}=8000, s_{3}=0, s_{4}=0$; (b) $n=6, \alpha=1, \beta=\frac{1}{50}, a=0, c=1, s_{0}=0, s_{1}=0, s_{2}=0, s_{3}=$ $1000000, s_{4}=0, s_{5}=0$. 

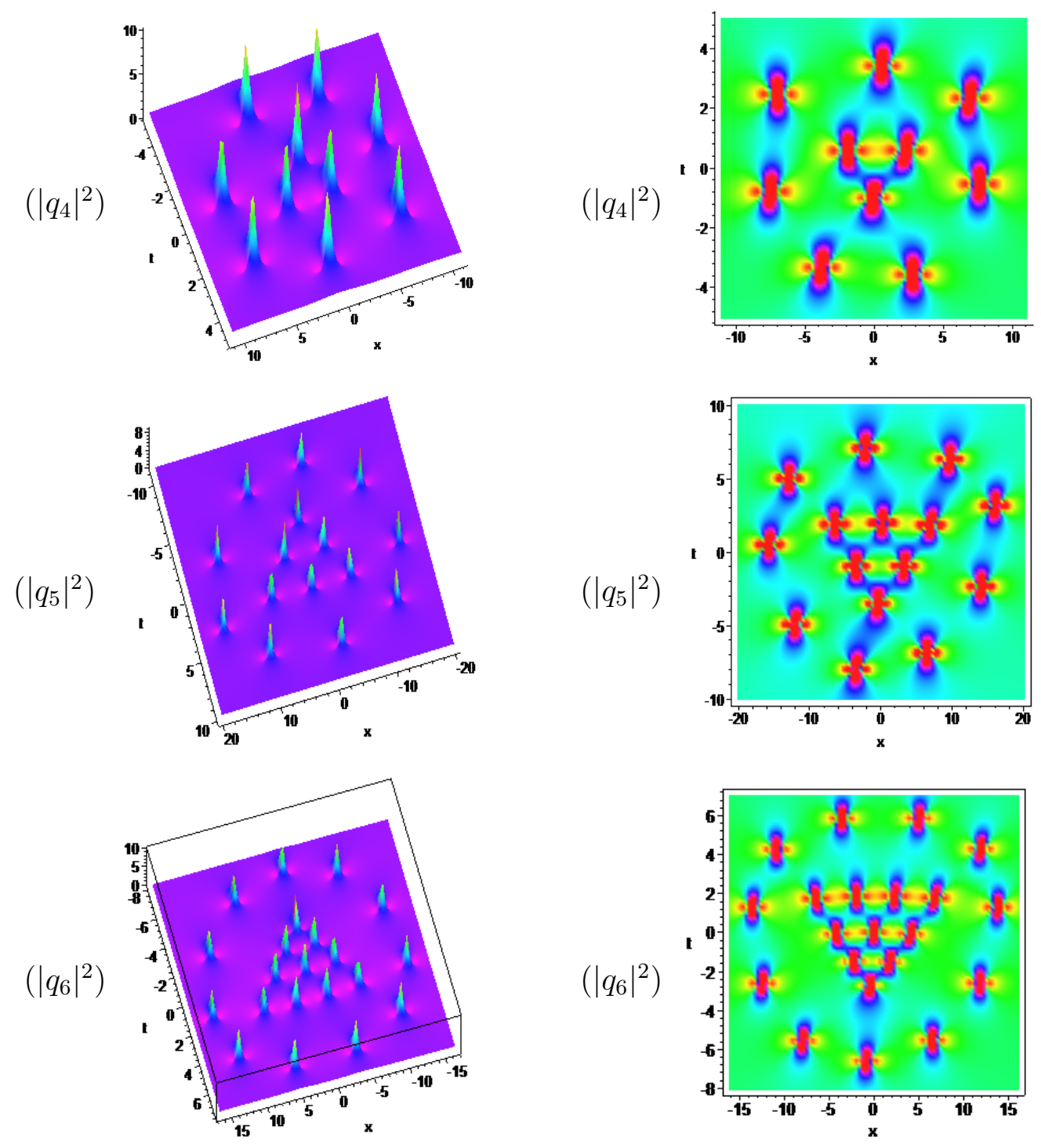

Figure 6. Ring plus triangular decomposition of $\left|q_{n}\right|^{2}$ (a) $n=4, \alpha=1, \beta=\frac{1}{50}, a=0, c=$ $1, s_{0}=0, s_{1}=10, s_{2}=0, s_{3}=10000$; (b) $n=5, \alpha=1, \beta=\frac{1}{50}, a=0, c=1, s_{0}=0, s_{1}=$ 50, $s_{2}=0, s_{3}=0, s_{4}=10000000$; (c) $n=6, \alpha=1, \beta=\frac{1}{50}, a=0, c=1, s_{0}=0, s_{1}=15, s_{2}=$ $0, s_{3}=0, s_{4}=0, s_{5}=10000000$. 

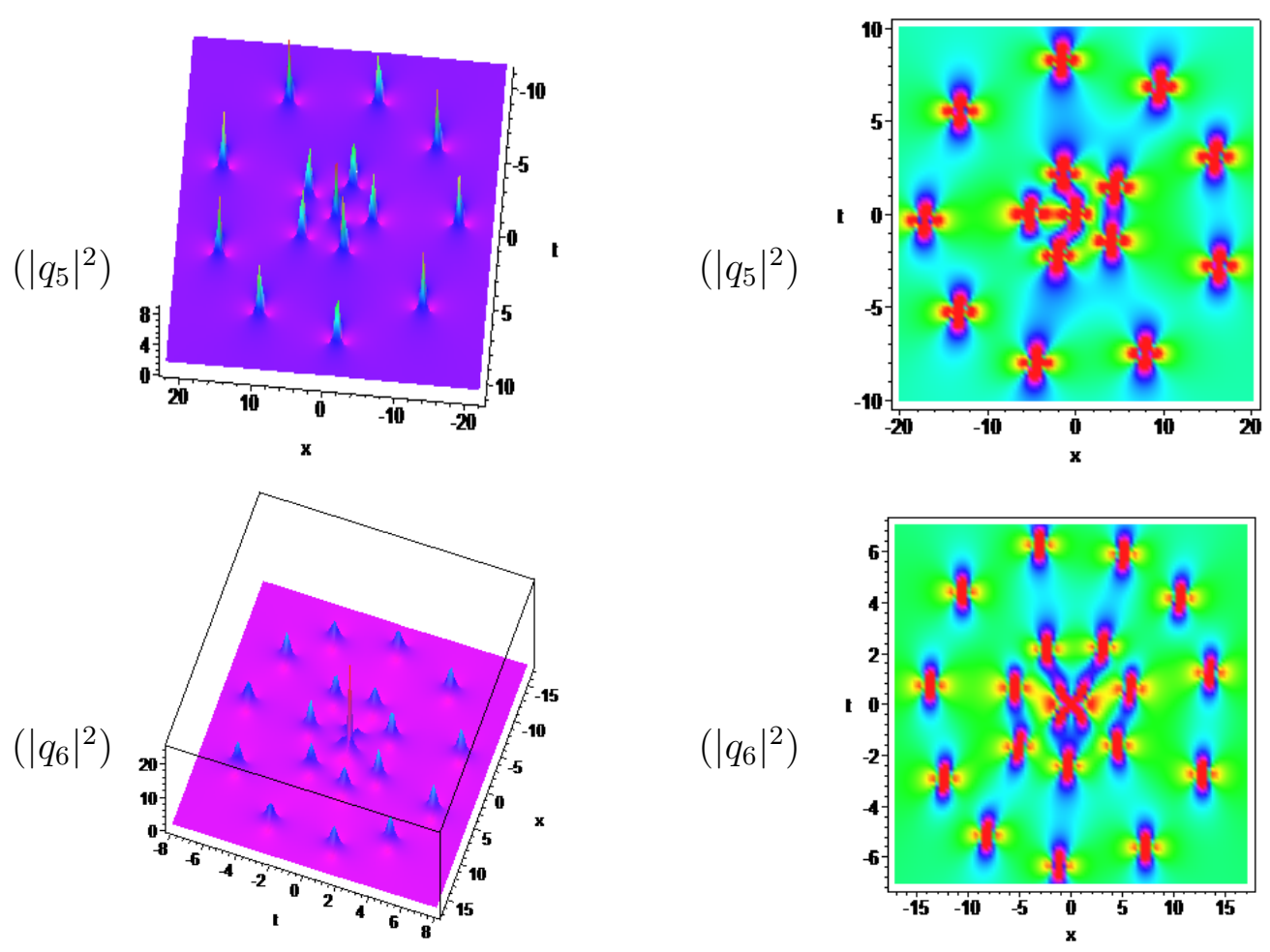

Figure 7. Fundamental pattern plus ring structure decomposition (a) $n=5, \alpha=1, \beta=$ $\frac{1}{50}, a=0, c=1, s_{0}=0, s_{1}=0, s_{2}=0, s_{3}=80000, s_{4}=20000000$; (b) $n=6, \alpha=1, \beta=$ $\frac{1}{50}, a=0, c=1, s_{0}=0, s_{1}=0, s_{2}=0, s_{3}=0, s_{4}=100000, s_{5}=10000000$.
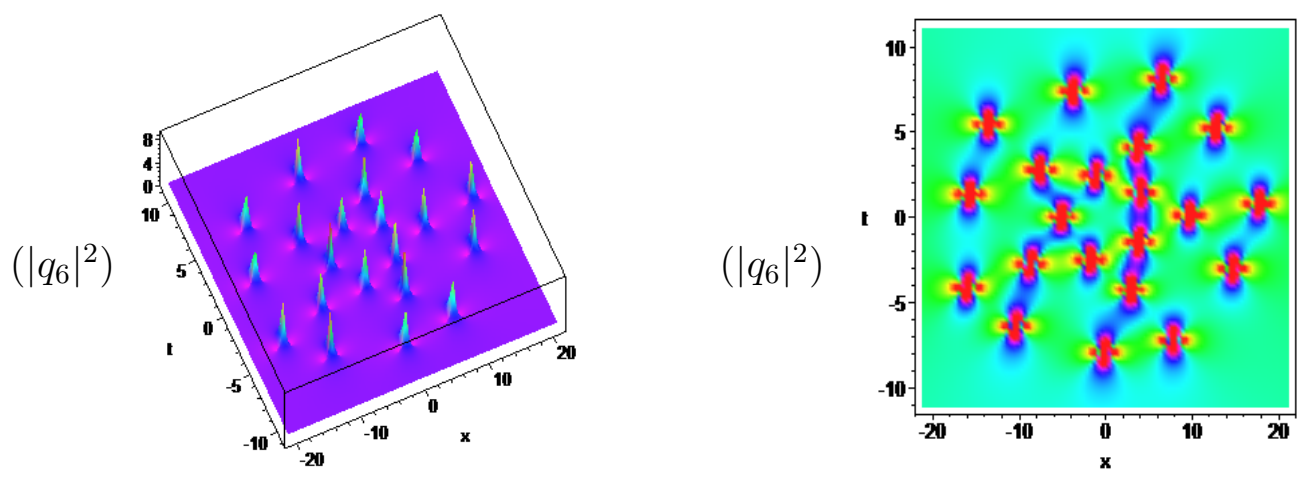

Figure 8. Complete decomposition of $\left|q_{6}\right|^{2}$ into rings of single peaks $\alpha=1, \beta=\frac{1}{50}, a=$ $0, c=1, s_{0}=0, s_{1}=0, s_{2}=1000, s_{3}=0, s_{4}=0, s_{5}=100000000$. 